\title{
A ERGONOMIA DO AMBIENTE CONSTRUÍDO EM SALAS DE FISIOTERAPIA PARA CRIANÇAS COM PARALISIA CEREBRAL: UMA REVISÃO SISTEMÁTICA
}

\author{
THE ERGONOMICS OF BUILT ENVIRONMENT IN PHYSIOTHERAPY \\ ROOMS FOR CHILDREN WITH CEREBRAL PALSY: A SYSTEMATIC \\ REVIEW
}

\author{
Marcella Lôbo ${ }^{1}$, Bach. \\ marcellavlobo@gmail.com e https://orcid.org/0000-0002-0558-678X \\ Vilma Villarouco ${ }^{2}$, D. Sc. \\ vvillarouco@gmail.com e https://orcid.org/0000-0002-4520-3683 \\ ${ }^{1}$ PPGDesign-UFPE, Recife, Brasil \\ ${ }_{2}^{2}$ PPGDesign-UFPE; PPErgo-UFPE; PPGAU+D-UFC, Recife, Brasil
}

\begin{abstract}
ambiente construído, sala de fisioterapia, paralisia cerebral, revisão sistemática
Este artigo apresenta uma revisão sistemática de literatura relacionada ao entendimento do estado da arte em relação aos cuidados com os ambientes de tratamento de pessoas com deficiência. $\mathrm{O}$ foco do interesse para a definição das palavras-chave da busca contempla a interação de crianças com paralisia cerebral e os ambientes de fisioterapia no qual se inserem, a fim de encontrar diretrizes que já tenham sido elaboradas e questões pesquisadas envolvendo a temática. Como metodologia, a revisão sistemática baseou-se no método Cochrane, através da exploração exaustiva da combinação de palavras-chave através portal de Periódicos CAPES, gerando um número de artigos significativo, onde as vertentes de estudo nos últimos cinco anos, puderam ser percebidas como diretamente ligadas aos aspectos imediatos do tratamento, sem relação da percepção do espaço. Os resultados encontrados demonstram uma lacuna no estudo da interação entre as crianças com paralisia cerebral e os ambientes de salas de fisioterapia por elas utilizados para o tratamento diário, contribuindo assim com a pesquisa final fundamentando uma justificativa concreta para o estudo.
\end{abstract}

\section{built environment, physiotherapy room, cerebral palsy, sistematic review}

This article presents a literature systematic review relating the state of art related with cares founded at the treatment environment to person with any disability. The interest focus for the key words definition ruled at the children's with cerebral palsy interaction and the physiotherapy rooms where they belong, to find the guidelines and questions that were already elaborated about it, evolving the theme. As a methodologic process, it was used the Cochrane method, through exhaustive exploration of key words combination in the Periodic CAPES portal, it was generated a meaningful number of articles which presents the strands of them, in the last five years, as immediately connected only with treatment aspects, without any relation with spaces perception. The results founded show a meaningful gap at the interaction study between children's with cerebral palsy and the environment used by them for theirs diary treatments, thus contributing with the final research substantiating a concrete justify for the study. 


\section{Introdução}

A Ergonomia vista como disciplina científica que envolve conhecimentos de diversas áreas é a base para a pesquisa em desenvolvimento que demandou a busca abordada nesse artigo. Nela foram previstos aspectos importantes da utilização do ambiente e todos os condicionantes relevantes a ele associados - que podem ser físicos, cognitivos, antropométricos, da acessibilidade, psicossociais e culturais - a fim de minimizar as possibilidades de uma insuficiência ainda mesmo na fase conceitual do projeto.

A Ergonomia do Ambiente Construído (EAC), como ramo recente da ergonomia voltado para a adequação das tarefas e ambientes às necessidades de seus usuários, acomoda os anseios e preocupações legítimos da relação humano-ambiente-atividade. A pesquisa aqui aludida insere-se nesta linha e investe em um afunilamento de disciplinas, conjugando design, ergonomia e arquitetura, com o intuito de identificar indicadores que possam colaborar no estabelecimento de diretrizes para ambientes que favoreçam o tratamento de usuários com paralisia cerebral.

Pesquisa anterior (Reabilit: estudo de caso em uma instituição assistencial de saúde no recife e a influência da iluminação nas atividades terapêuticas) que analisou o ambiente construído para o tratamento de reabilitação motora e mental identifica que é de suma importância, para um aumento na capacidade de absorção cerebral das informações, uma correta organização do ambiente de atendimento, facilitando a dinâmica do terapeuta e a compreensão da atividade pelo paciente em questão. Ou seja, a usabilidade tem influência direta na relação paciente-espaço de tratamento.

A problemática é estabelecida através da busca a respostas ao questionamento: quais elementos relacionados à ergonomia do ambiente construído têm influência direta e positiva na estimulação do tratamento terapêutico diário de paciente com paralisia cerebral? Com uma contribuição social relevante, a pesquisa se faz necessária trazendo benefícios mensuráveis e reais para tais usuários, mas também aos profissionais que lidam com os tratamentos fisioterápicos e aos projetistas que proveem os ambientes para tais atividades

Nesse contexto, o tema da pesquisa se fundamenta na estimulação proporcionada pelo ambiente construído como interferência direta no desenvolvimento de atividades terapêuticas de cunho diário em pacientes com paralisia cerebral, a fim de observar suas respostas e concluir quais os elementos significativos que impulsionam positivamente o tratamento diário desses pacientes.

A revisão sistemática da literatura conduzida foi estruturada relacionando palavras-chave do tema de pesquisa com elementos específicos do ambiente construído, no sentido de aprimorar a relação entre a EAC e o tema abordado na pesquisa aludida, auxiliando no entendimento de questões conceituais e suas conexões com o estudo. Conforme a combinação das palavras chave adotadas, os resultados desta revisão trazem achados que identificam o estado da arte em pesquisas que relacionam elementos de ambientes com o tratamento fisioterapêutico de pacientes com deficiências, não se limitando apenas aos estudos envolvendo a que está focada na pesquisa.

\subsection{Paralisia Cerebral}

A Paralisia Cerebral (PC), também conhecida como encefalopatia crônica da infância, é uma doença não progressiva e pode ser definida como "um distúrbio permanente, embora não invariável, do movimento e da postura, devido a defeito ou lesão não progressiva do cérebro no começo da vida" (FERRARETTO E SOUZA, 1998). O termo foi proposto em 1897 por Freud, tendo sido posteriormente consagrado por Phelps.

Na ocorrência da PC, uma alteração dos movimentos motores, causada por lesões, danificações permanentes ou disfunções relacionadas ao sistema nervoso central (SNC) é a característica classificatória no diagnóstico final. As causas podem ocorrer no período inicial gestacionário (malformações congênitas), durante o parto (falta de oxigenação cerebral ou uso do fórceps) ou no início da infância (infecções cerebrais). 
Por não ser uma doença progressiva, a PC não evolui com o passar do tempo, entretanto algumas características podem se transformar com o desenvolvimento e crescimento corporal nesses pacientes, é o caso do estirão do crescimento capaz de alterar a estrutura corpórea da criança a partir dos 8 (oito) ou 9 (nove) anos, podendo causar compressão de órgãos ou deficiências ósseas em crianças com PC, gerando pioras significativas no quadro estacionário.

Segundo a Organização Mundial de Saúde (OMS), em 2011, 5,1\% (cinco vírgula um por cento) das crianças com idade entre zero e quatorze anos possuem algum tipo de incapacidade, sendo a PC uma das deficiências mais comuns causadoras de incapacidades infantis. Ou seja, 1,5 a 2,5 a cada 1000 partos de fetos nascidos vivos são diagnosticados com PC nos níveis moderada e severa (GUPTA; APPLETON, 2001). O diagnóstico costuma ser, em média, observado no terceiro mês após o nascimento, fase em que o bebê costuma apresentar sinais de prontidão e desenvolvimento corporal, que não costumam ser percebidos até esta fase.

Para a comunidade médica, a PC pode ser classificada de acordo com dois critérios, pelo quadro clínico apresentado em cada tipo de disfunção motora, ou pela localização da disfunção motora. Na primeira abordagem são divididos em extrapiramidal ou discinético (atetóide, coréico e distônico), atáxico, misto e espástico; na segunda, em quadriplegia, diplegia e hemiplegia.

A Quadriplegia atua em todos os membros do paciente, sendo comumente associada a microcefalia, deficiência mental e epilepsia. A Diplegia é a deficiência dos membros inferiores. A Hemiplegia é o comprometimento dos membros superiores. A Discinesia é mais rara, uma vez que se observa através de movimentos involuntários. A Ataxia também se enquadra na raridade, apresenta tremores intencionais, alterações de equilíbrio ou coordenação. $O$ tipo Misto é a associação das duas classificações (por exemplo a ataxia com a plegia), esse é o caso mais comum (FERRARETTO E SOUZA, 1998).

Dentro dos quadros apresentados, a PC também pode vir associada com outros diagnósticos colaterais, que não ocorrem em todos os pacientes, mas podem vir como conjunto para muitos. É o caso da deficiência mental, epilepsia, distúrbios de linguagem, deficiência visual, distúrbios de comportamento e deficiências ortopédicas, todos se relacionam com as alterações sofridas nas áreas cerebrais específicas para cada paciente.

O tratamento é voltado para uma qualidade de vida obtida através da fisioterapia (e outras terapias associadas) e ao desenvolvimento motor. Cada criança deve ser avaliada individualmente proporcionando um método mais eficiente para cada época do seu crescimento. A fisioterapia tem função direta para estes pacientes pois atua na inibição da atividade reflexa anormal normalizando o tônus muscular e assim facilitando o movimento normal do corpo, otimizando a função e reduzindo a incapacidade. Os estudos medicinais indicam que os alongamentos músculotendinosos devem ser lentos e realizados diariamente para manter a amplitude de movimento e reduzir o tônus muscular (FERRARETTO E SOUZA, 1998)

As intervenções fisioterapêuticas podem ser subdividas em quatro podendo ser combinadas entre si a fim de potencializar o desenvolvimento da criança. $\mathrm{O}$ enfoque biomecânico (movimento, resistência e as forças necessárias para as atividades cotidianas), neurofisiológico, do desenvolvimento (conhecidos juntos como neuroevolutivo, combinando técnicas neurofisiológicas e de tratamentos específicos como o Bobath) e sensorial (técnicas com experiências sensoriais).

O futuro dessas crianças também sofre influência quando observados o grau de deficiência mental, o número de crises epilépticas e a intensidade do distúrbio de comportamento, tornando os resultados obtidos com tratamento praticamente ínfimos quanto à evolução da deficiência. Dessa forma, o fisioterapeuta deve estar em constante atualização a fim de garantir eficácia em sua intervenção diária com esses pacientes. Em alguns casos, um programa de reabilitação física deverá atentar a outros fatores que não somente ao 
desenvolvimento motor, sendo necessários atos específicos como levantar-se, pegar objetos, etc., aprimorando as funções existentes em cada um dos assistidos.

\subsection{Salas de Fisioterapia}

As instituições de saúde para tratamento de fisioterapia são classificadas como Estabelecimento Assistencial de Saúde (EAS) por se tratarem de um estabelecimento destinado "à prestação de assistência à saúde à população, que demande o acesso de pacientes, em regime de internação ou não, qualquer que seja o seu nível de complexidade". (RDC 50, 2002, p.137). Dada essa colocação, o ambiente relacionado ao presente estudo segue algumas normativas presentes na legislação brasileira, municipal, estadual e local.

Segundo a cartilha do Ministério de Saúde - Normas para Projetos Físicos de Estabelecimentos Assistenciais de Saúde (1994) - Portaria MS nº 1.884, as clínicas de fisioterapia se enquadram como Serviço de Referência em Medicina Física e Reabilitação, cujo objetivo é:

Prestar assistência intensiva em reabilitação aos portadores de deficiência física, referenciados por outros serviços de saúde, constituindo-se na referência de alta complexidade em reabilitação (motora e sensório motora), de acordo com os princípios definidos pela NOAS-SUS 01/2001, devendo integrar-se a uma rede regionalizada e hierarquizada de assistência ao portador de deficiência física.

Sobre os ambientes físicos a norma indica aspectos específicos que devem ser seguidos, conforme a tabela 1. Além dos parâmetros mínimos de dimensão, também são abordados os materiais para piso e paredes como sendo obrigatórios de cor clara, apresentar resistência, se impermeável e lavável. Além de alguns equipamentos necessários para o funcionamento da instituição.

Tabela 1: Unidade Funcional 5 Apoio ao Diagnóstico e Terapia (ADE, HF, HQ, ED)

\begin{tabular}{l}
\hline UNIDADE FUNCIONAL: 5 - APOIO AO DIAGNÓSTICO E TERAPIA \\
\hline \multicolumn{1}{|c|}{ UNIDADE / AMBIENTE } \\
\cline { 5 - 6 }
\end{tabular}

Fonte: Ministério de Saúde - Portaria MS nº 1.884, 1994.

Em trabalho desenvolvido com o objetivo de avaliar a influência das cores e da iluminação dos ambientes de tratamento fisioterápico de pacientes com PC (Reabilit: estudo de caso em uma instituição assistencial de saúde no recife e a influência da iluminação nas atividades terapêuticas) constatou, através de um experimento durante as sessões diárias, que a presença/ausência de luz e a alteração de suas cores foi percebida e trouxe alterações motoras durante o período da atividade, causando, ora facilidade no manuseio dos membros por meio do profissional, ora dificuldade, contribuindo na recepção dos exercícios por estes pacientes. 


\subsection{Ergonomia do Ambiente Construído}

A ergonomia como disciplina foi constituída a partir do século XX como um estudo sistêmico da relação homem-máquina-trabalho. Já a Ergonomia do Ambiente Construído (EAC) busca focar na adequação dos ambientes às atividades nele desenvolvidas sempre atentando às necessidades dos usuários.

Uma vez que o espaço construído é bem projetado, segundo Vergara e Rodrigues (2018), sua apropriação pode gerar um aumento na competência dos usuários, sendo necessárias decisões projetuais que tenham base, inclusive em sua observação inicial, e que sejam pautadas no usuário do ambiente em questão.

A ergonomia aponta que os ambientes devem ser projetados de forma eficiente relacionando sempre o espaço com as atividades a serem desenvolvidas. Logo, a Ergonomia do Ambiente Construído se constitui como uma ferramenta de apoio ao projetista, e consequentemente ao usuário, para que suas técnicas e métodos possam servir como ponto de partida na concepção de espaços mais integrativos e capazes de impulsionar os que irão desfrutar de suas dependências.

Torna-se essencial para uma melhor configuração do ambiente, um estudo aprofundado das atividades exercidas no espaço associado aos conceitos e conhecimentos da ergonomia nos projetos arquitetônicos e de design de interiores propostos. Os elementos que fazem parte da observação espacial sob a ótica da ergonomia "são aqueles referentes ao conforto ambiental (lumínico, térmico e acústico), à percepção ambiental (aspectos cognitivos), adequação de materiais (revestimentos e acabamentos), cores e texturas, acessibilidade, medidas antropométricas (layout, dimensionamento), e sustentabilidade" (VILLAROUCO; MONT'ALVÃO, 2011, p. 31).

Para aplicar esses conceitos à observação dos espaços, a avaliação deve ser pautada em duas vertentes, uma de cunho físico e outra de cunho psicológico (onde entram a percepção do usuário em relação ao ambiente em questão). Segundo Mont'Alvão (2015), na "tarefa, e a relação do usuário com o meio, desenvolve-se a Ergonomia do Ambiente Construído, a fim de projetar um ambiente adequado aos requisitos ergonômicos".

Dessa forma, é premissa da Ergonomia do Ambiente Construído identificar a combinação do usuário com o espaço e seus componentes, constatando a interferência deste na atividade daquele, uma vez que ambos constituem uma parte de um mesmo sistema, não podendo serem compreendidos de forma isolada.

\section{Metodologia}

A Revisão Sistemática da Literatura (RSL) vem sendo um importante instrumento para a compilação de estudos desenvolvidos sobre um determinado tema que se deseja pesquisar, auxiliando na atualização do estado da arte e apresentando os elementos relevantes e significativos à pesquisa em questão.

A RSL possui princípios e etapas que foram seguidos, como critérios de inclusão e exclusão (que serão especificados ao longo do trabalho), e a sistematização dos dados encontrados em tabelas e quantitativos permitindo a certificação do estudo.

O método adotado para a realização dessa pesquisa foi baseado na RSL Cochrane desenvolvido pela Cochrane Collaboration (http://www.cochrane.org/ ou http://brazil.cochrane.org/) cuja intenção é a de obter as publicações mais relevantes sobre determinado tema ou assunto. A partir deste processo qualquer pesquisador se torna capaz de validar, refutar ou continuar com a pesquisa. É fundamentalmente constituído por três fases, apresentados na figura 1 . 


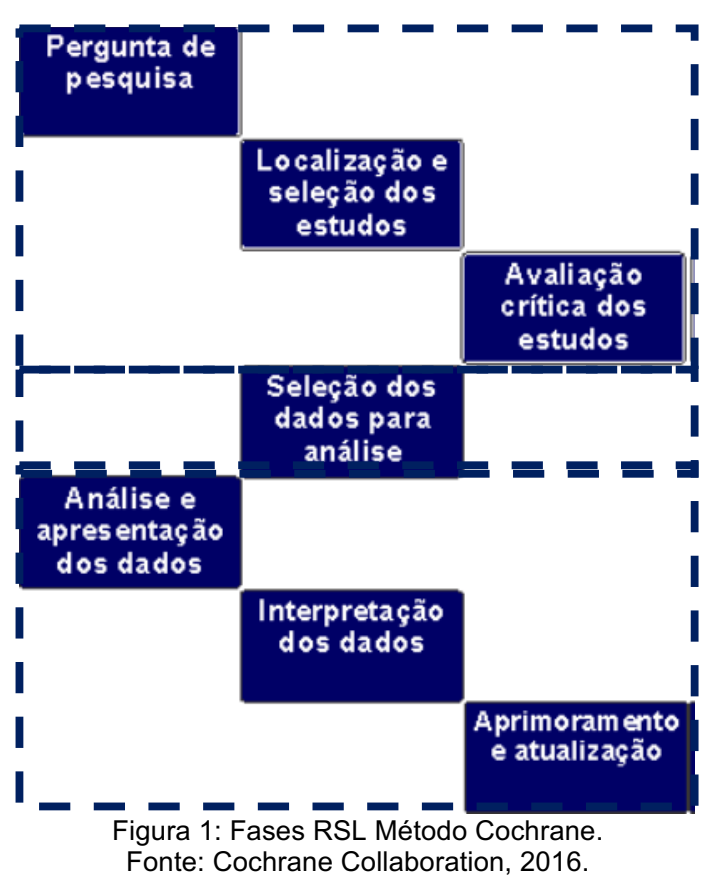

Estas fases compreendem os seguintes passos:

FASE 1: Planejamento e Formalização

a) Definição da questão ou problema de pesquisa;

b) Definição dos objetivos da pesquisa (geral e específicos);

c) Definição das palavras-chave, assim como seus sinônimos;

d) Definição dos critérios de inclusão e exclusão;

e) Escolha da base de dados.

FASE 2: Condução e Execução do Protocolo

a) Calibração da busca;

b) Execução da busca;

c) Download dos dados das publicações;

d) Catalogação das publicações;

e) Seleção das publicações: Teste de relevância 1 (avaliação dos elementos pré e pós textuais - título, resumo e conclusão) e Teste de relevância 2 (leitura completa dos artigos selecionados)

FASE 3: Sumarização

a) Tabular os dados obtidos;

b) Analisar os resultados obtidos;

c) Condensar e publicar resultados.

Pelo desenvolvimento da pesquisa, foi executada a RSL apresentada no presente trabalho explorando artigos internacionais relacionados aos elementos do ambiente construído, sendo conduzida a partir do Portal de Período CAPES, pela adequação indicada na literatura especializada, sempre associado à ferramenta de Busca Avançada. Como recorte temporal, foram pesquisados artigos, publicados nos últimos cinco anos (2015-2020), na língua inglesa e adotando o critério de serem revisados por pares. Pela grande quantidade de resultados obtidos pela combinação de palavras chave, foram analisadas as cinquenta primeiras ocorrências por critério de relevância.

A pergunta norteadora da pesquisa foi a seguinte: Com relação aos aspectos da ergonomia do ambiente construído, em que condições se encontram as pesquisas voltadas para os pacientes com paralisia cerebral e sua interação com os espaços de terapia? As palavras-chave foram selecionadas conforme o tema (physiotherapy room; physiotherapy clinic; physiotherapy; physical therapy; rehabilitation NOT drugs; 
cerebral palsy) e direcionados para ergonomia do ambiente construído (human factors, environment interaction; environment influence; built environment; environment enrichment; ergonomic).

Diante do exposto, foi feito o cruzamento de todas elas e foram relacionados os artigos encontrados com os países de criação do estudo. Foram encontrados 7.376 artigos no total conforme apresentado na tabela 2.

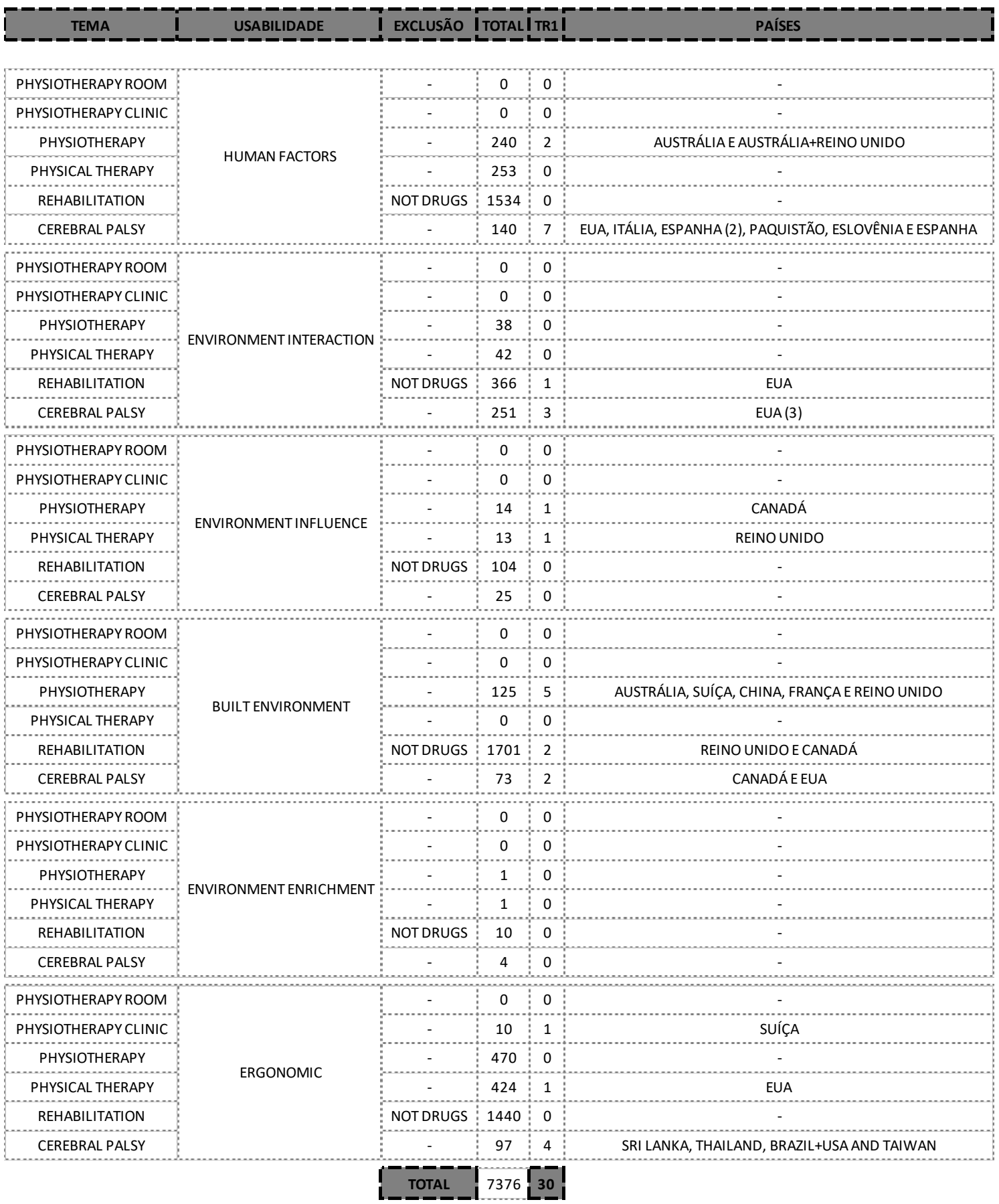

Tabela 2: Revisão Sistemática de Literatura

Fonte: Autoras (2020) 
No primeiro teste de relevância, os títulos, resumos e conclusões foram observados, excluindo aqueles que não se enquadravam no tema. No teste seguinte, foram lidos na íntegra todos os remanescentes, sendo observados trinta artigos ao final do teste de relevância 1 (TR1) apresentado na tabela 3, conforme legenda.

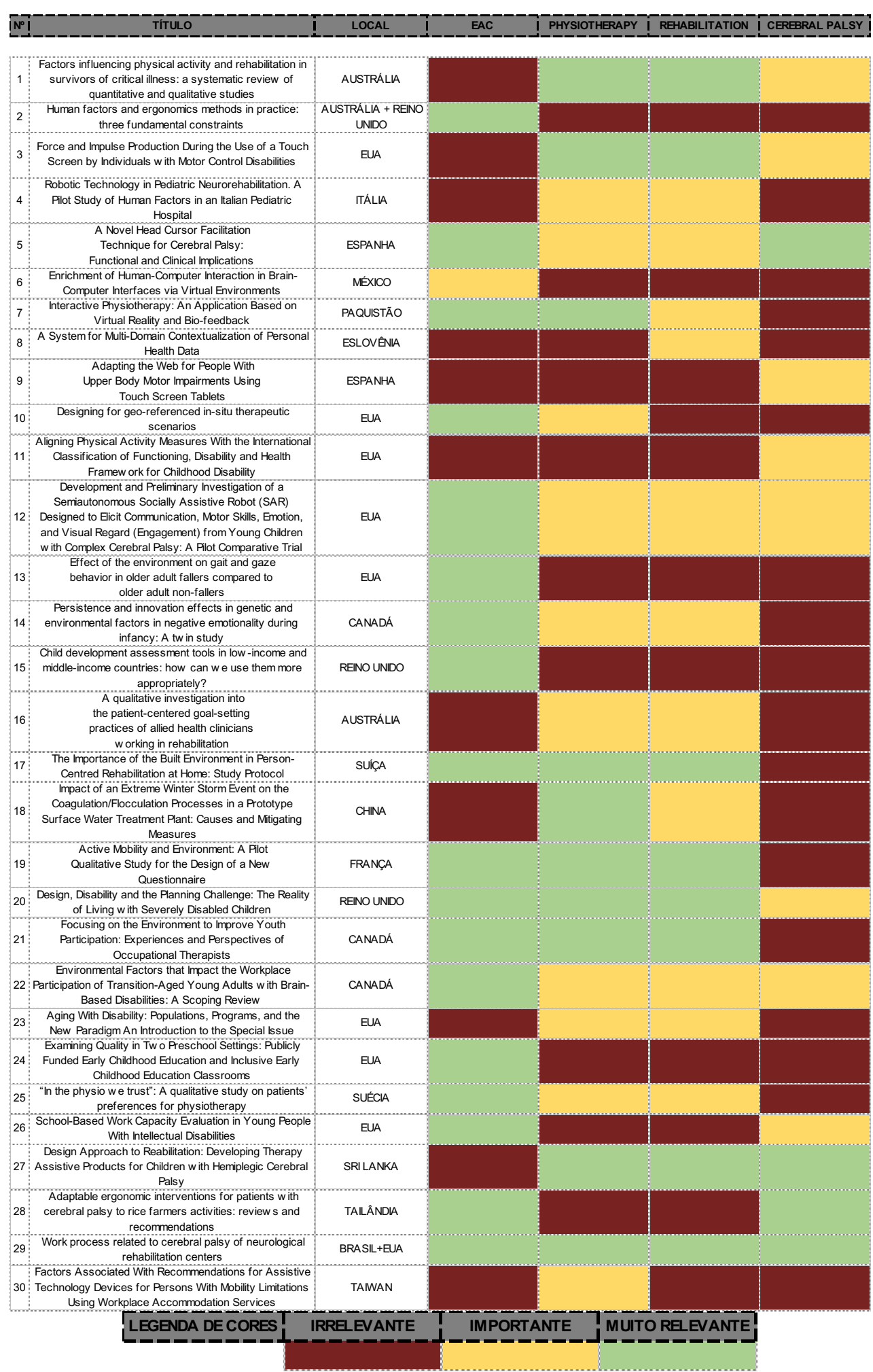

Tabela 3: Teste de Relevância 1

Fonte: Autoras (2020) 
No segundo teste de relevância, ficaram os artigos com maior pertinência para a temática, sendo lidos na íntegra todos os remanescentes e escolhidos os seis com maior relevância para o estudo ergonomia do ambiente construído contribuindo para a pesquisa proposta. Os seis artigos selecionados foram aprofundados em resenhas individuais que contribuirão para o desenvolvimento da pesquisa sobre a influência de ambientes para o tratamento fisioterápico de pacientes com paralisia cerebral, como mencionado anteriormente.

\section{TESTEDERELEVÂNCIA 2}

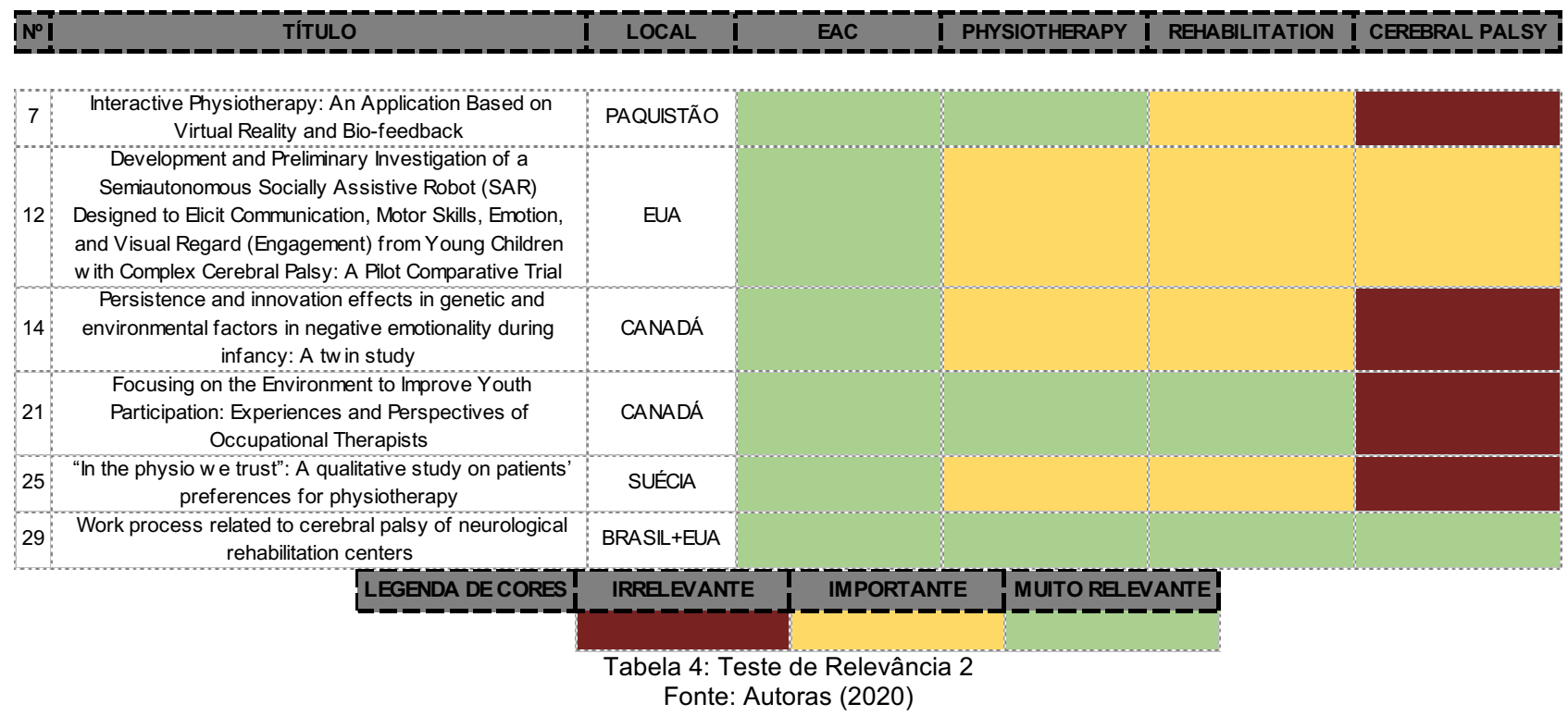

\section{Resultados}

Foram analisados os seis artigos mais relevantes encontrados na RSL, originados em países como Paquistão, EUA, Canadá e Suécia. Tendo em vista os estudos selecionados, observou-se um maior aprofundamento da literatura nos países norte-americanos. Identificando os campos de atuação relacionados às pesquisas apresentadas, algumas abordagens foram voltadas para o ambiente construído e sua influência na análise do usuário. As considerações estão no presente capítulo.

As obras consideradas relevantes para o trabalho encontram-se identificadas a seguir, com seus conteúdos apresentados sistematicamente.

O primeiro artigo apresenta critérios relevantes quanto ao uso da ferramenta de realidade virtual no desempenho de pacientes fisioterápicos associados ao ambiente simulado. 


\begin{tabular}{|c|c|}
\hline ANO & 2018 \\
\hline TíTULO & $\begin{array}{c}\text { Interactive Physiotherapy: An Application Based on Virtual Reality and } \\
\text { Bio-feedback }\end{array}$ \\
\hline AUTORES & $\begin{array}{c}\text { Attiya Baqai } \\
\text { Khuhed Memon } \\
\text { Azam Rafique Memon } \\
\text { Syed Muhammad Zaigham Abbas Shah }\end{array}$ \\
\hline OBJETINO & $\begin{array}{l}\text { Desenvolver um sistema integrado combinando a fisioterapia com o bio- } \\
\text { feedback e a realidade virtual. }\end{array}$ \\
\hline MÉTODOS & $\begin{array}{l}\text { Desenvolvimento de uma sistema de telereabilitação para pacientes e } \\
\text { fisioterapeutas. }\end{array}$ \\
\hline RESULTADOS & $\begin{array}{l}\text { O softw are testado indicou preferências de realidade virtual baseado } \\
\text { em exercícios de fisioterapia opostos ao normal. Durante os testes, } \\
\text { foram observados erros em medidas fisiológicas nos sensores } \\
\text { biológicos, entretanto trouxeram efeitos insignificantes no progresso do } \\
\text { regimento do jogo. }\end{array}$ \\
\hline PUBLICAÇÃO & Wireless Pers Commun \\
\hline LOCAL & Sindh, Paquistão \\
\hline
\end{tabular}

O foco encontra-se no tema fisioterapia trabalhado através do auxílio da realidade virtual (Virtual Reality VR) apresentando um comparativo com portadores da doença de Parkinson (Parkinson's Desease - PD). A PD é uma disfunção neurológica que afeta os movimentos motores causando, como ênfase, tremores involuntários. Neste trabalho a VR tem a intenção de replicar um ambiente seguro enquanto simula a presença do usuário no espaço, permitindo sua interação com ele.

Um sistema integrado entre bio-feedback e realidade virtual, é o objetivo desse trabalho, observado a partir da evolução da VR, informações do equipamento e uma metodologia voltada para o ambiente de fisioterapia onde os usuários realizam exercícios de acordo com os componentes voltados para o tratamento.

Considerando que as rotinas de comando de exercícios, pautadas em métodos convencionais, podem proporcionar exaustão e desinteresse para os pacientes, jogos foram desenvolvidos, a fim de modificar a dinâmica usualmente adotada através da VR.

No teste aplicado com quatro voluntários, sendo uma mulher e dois homens saudáveis com idades entre vinte e seis e trinta e seis anos, e uma mulher de sessenta e oito anos diagnosticada com a doença de Parkinson, os sinais vitais foram observados através do sensor e-health v2.0, as respostas físicas por um dispositivo de webcam que permite a visualização de imagem a cores, conhecido como Microsoft Kinect, um óculos VR, chamado de Oculus Rift que permite a VR imersiva e um console Unity 5.5.0.

Os resultados indicaram que os parâmetros fisiológicos permaneceram normais durante a sessão concluindo que o software é uma eficiente e interessante ferramenta para fisioterapia em ambos os pacientes estudados. Os testes apontaram que os sujeitos preferiram a VR ao formato normal, ainda que tenham sido observados alguns erros nas medições dos biossensores. $\mathrm{O}$ aplicativo desenvolvido também pode ser acrescido para paraplegia através da configuração da propriedade de postura corporal, além da adição de biossensores baseados na performance dos pacientes. Obtiveram um comprometimento e desempenho maior com os exercícios propostos e uma interação maior com o ambiente virtual, proporcionando uma atividade com benefícios maiores para os usuários, contribuindo para o aumento na utilização da ferramenta em tratamentos fisioterápicos.

O segundo artigo apresentado aborda uma investigação sobre a interação entre um robô projetado para auxiliar crianças com severa paralisia cerebral e suas atividades. 


\begin{tabular}{|c|c|}
\hline ANO & 2019 \\
\hline TÍTULO & $\begin{array}{l}\text { Development and Preliminary Investigation of a Semiautonomous Socially } \\
\text { Assistive Robot (SAR) Designed to Elicit Communication, Motor Skills, } \\
\text { Emotion, and Visual Regard (Engagement) from Young Children w ith } \\
\text { Complex Cerebral Palsy: A Pilot Comparative Trial }\end{array}$ \\
\hline AUTORES & $\begin{array}{c}\text { Cecilia Clark } \\
\text { Levin Sliker } \\
\text { Jim Sandstrum } \\
\text { Brian Burne } \\
\text { Victoria Haggett } \\
\text { Cathy Bodine }\end{array}$ \\
\hline OBJETIVO & $\begin{array}{l}\text { Desenhar um sistema autônomo que possa responder apropriadamente } \\
\text { à necessidade da criança no contexto clínico com objetivo final de criar } \\
\text { informações e proporcionar melhorias nas interações terapéuticas. }\end{array}$ \\
\hline MÉTODOS & $\begin{array}{l}\text { Cruzamento de métodos de design comparando um controle condicional } \\
\text { com uma condição experimental (protótipo robótico). }\end{array}$ \\
\hline RESULTADOS & $\begin{array}{c}\text { Crianças com paralisia cerebral complexas apresentaram forte } \\
\text { engajamento e aspectos quantitativos e qualitativos de engajamento que } \\
\text { poderão ser aplicados futuramente. }\end{array}$ \\
\hline PUBLICAÇÃO & Hindaw i Advances in Human-Computer Interaction \\
\hline LOCAL & Denver, EUA \\
\hline
\end{tabular}

Este artigo foi desenvolvido como passo inicial para a criação de um "Robô Socialmente Assistente" (Socially Assistive Robots - SAR) completamente autônomo que possa ser utilizado de forma terapêutica em crianças com paralisia cerebral. O primeiro passo foi determinar as atividades e interesses que atraiam a população trabalhada e em seguida verificar a aceitabilidade do SAR como uma espécie de brinquedo para os usuários testados. A finalidade seria estimular o progresso cognitivo e físico além das habilidades sociais dos pacientes.

O público alvo foi de oito participantes com limitações de postura e descontrole de movimentos, variando entre dezoito meses e cinco anos de idade. Foram apresentadas duas ações principais: interagir com o brinquedo adaptado e em seguida, interagir com o SAR para os mesmos movimentos num total de seis sessões individuais, cada uma com duração de trinta minutos.

$\mathrm{O}$ estudo completou sete visitas por participantes durante doze semanas. O experimento inicial contou ativamente com a participação dos terapeutas ocupacionais junto aos pais. Após as visitas experimentais, o investigador aplicava um checklist que determinava a saúde, o humor e o nível intelectual da criança. Foram coletados vídeos de cada sessão e analisados através do método Morae.

Os investigadores que observaram os vídeos notaram a ausência de habilidade de compressão nos pacientes. Dentre eles, cinco dos oito sujeitos tiveram um nível de engajamento considerado de excelente adaptação. Ficou evidente que os sujeitos se tornaram crescentemente confortáveis com o SAR a cada sessão.

Apresentando dados quantitativos relevantes, o artigo demonstra que a tecnologia de interação com objetos de interesse dos pacientes pode ser utilizada em terapias correntes com um alto índice de resposta das crianças com PC. Dessa forma, pretende-se expandir o conhecimento para as características físicas e psicológicas do ambiente, capazes de proporcionar uma maior integração entre os pacientes abordados e os espaços observados. 
O terceiro artigo apresenta o papel da interferência dos fatores ambientais nas experiências e emoções consideradas negativas durante a infância, a partir de um comparativo entre estudos.

\begin{tabular}{|c|c|}
\hline ANO & 2017 \\
\hline TITULO & $\begin{array}{c}\text { Persistence and innovation effects in genetic and environmental factors } \\
\text { in negative emotionality during infancy: A tw in study }\end{array}$ \\
AUTORES & $\begin{array}{c}\text { Lyndall Schumann } \\
\text { Michel Boivin } \\
\text { Stephane Paquin } \\
\text { Eric Lacourse } \\
\text { Mara Brendgen } \\
\text { Frank Vitaro } \\
\text { Ginette Dionne } \\
\text { Richard E. Tremblay } \\
\text { Linda Booi }\end{array}$ \\
OBJETIV & Estimar genética e ambientalmente as contribuições individuais da \\
infância emocionalmente negativa.
\end{tabular}

O objetivo do artigo foi criar um estudo longitudinal, a fim de estimar o cruzamento de aspectos genéticos e as interferências ambientais observadas entre os cinco e dezoito meses de vida, analisando as emoções negativas e sua dissolução a longo prazo. Gêmeos foram recrutados desde o nascimento na província do Quebec. A escala emocional de negatividade foi composta de sete características utilizadas como componentes do instrumento. A medição abordou dificuldade, raiva e confusão como principais aspectos para a observação dos usuários estudados.

A pontuação para o teste variava de um (baixa negatividade) a sete (alta negatividade). O estudo resultou em pontuações semelhantes para meninos e meninas demonstrando influência do ambiente na vida cotidiana dos usuários. E os dados eram obtidos de forma comparativa entre os gêmeos, que recebiam o mesmo estímulo, e sua percepção individual. No total, foram seiscentos e trinta e oito pares de gêmeos analisados durante a pesquisa.

Nos primeiros dois anos de vida, fatores genéticos e ambientais foram considerados essenciais na emocionalidade negativa, pois a evidência indicou que essa essência negativa é desenvolvida através da dinâmica do usuário com o ambiente. Os sujeitos que alcançaram níveis altíssimos de negatividade apresentaram problemas como depressão, ansiedade e agressão em crianças ou adultos. Ou seja, aqueles que tiveram experiências negativas em relação ao ambiente explorado, foram mais negativos quando relacionados ao perfil emocional.

As características genéticas também foram apontadas como marcantes no comportamento final dos participantes (entre 40 e 48\%). Dessa forma, as atividades e sua relação com o espaço são consideradas a partir de uma fusão dos fatores genéticos com as experiências positivas ou negativas do espaço no qual os participantes se inserem. 
$\mathrm{O}$ artigo seguinte apresenta a perspectiva dos terapeutas ocupacionais e o progresso de jovens pacientes baseado na experiência do usuário com o ambiente.

\begin{tabular}{|c|c|}
\hline ANO & 2015 \\
\hline TÍTULO & $\begin{array}{l}\text { Focusing on the Environment to Improve Youth Participation: } \\
\text { Experiences and Perspectives of Occupational Therapists }\end{array}$ \\
\hline AUTORES & $\begin{array}{l}\text { Dana Anaby } \\
\text { Mary Law } \\
\text { Rachel Teplicky } \\
\text { Laura Turner }\end{array}$ \\
\hline OBJETIV & $\begin{array}{l}\text { Aprofundar conhecimentos do processo, usabilidade e eficácia da } \\
\text { intervenção terapéutica através das perspectivas dos terapeutas. }\end{array}$ \\
\hline MÉTODOS & $\begin{array}{l}\text { Uma intervenção qualitativa baseada no estudo do design, através de } \\
\text { uma ferramenta de entrevista conduzida por } 4 \text { (quatro) semanas. }\end{array}$ \\
\hline RESULTADOS & $\begin{array}{l}\text { Terapeutas preconcebem ideias sobre serviços emergenciais através } \\
\text { do desafio natural da intervenção. }\end{array}$ \\
\hline PUBLICAÇÃO & International Journal of Environmental Research and Public Health \\
\hline LOCAL & Montreal, Canadá \\
\hline
\end{tabular}

A participação dos jovens em casa, na escola e na comunidade é uma importante saída nas intervenções de reabilitação. Para este estudo, foi desenvolvido e testado um dispositivo intitulado Pathways and Resources for Engagement and Participation (PREP) que auxilia na participação dos jovens na comunidade, modificando aspectos do ambiente para sua melhor adaptação. Foi aplicado em jovens de doze a dezoito anos que possuíam dificuldades funcionais.

Esse dispositivo foi testado através de um estudo qualitativo e aplicado após modificações interventivas para validar o design empregado no produto final. Para este teste, doze terapeutas ocupacionais foram entrevistadas sobre as escolhas acerca das intervenções. A entrevista foi semiestruturada com cinco questões abertas ao final, apresentando as impressões e experiências das terapeutas.

Das participantes, dez possuíam mestrado e experiência clínica variando entre seis meses e vinte anos. Elas apontaram que as barreiras ambientais influenciavam nas particularidades dos pacientes, tendo sido apresentados como negativos ao longo das entrevistas elementos como: temporalidade do ambiente, adaptabilidade e localização geográfica.

Os aspectos sociais do ambiente, incluindo o suporte familiar e a amizade, foram apresentados como barreiras e/ou suporte na participação. Toda a família foi identificada como unidade para o processo de intervenção.

Os terapeutas desenvolveram uma solução baseadas em estratégia que minimizam as barreiras e fortificam as qualidades, através da individualização e do atendimento centrado no paciente. As estratégias e soluções de problemas também foram utilizadas, a fim de enfatizar aspectos de personalidade e participação. A percepção sobre o PREP demonstrou que os terapeutas apresentavam algumas dificuldades acerca do desafio de intervenção através de um mecanismo específico.

A pesquisa apresentou alguma dificuldade quanto a atual rotina da clínica onde foi implementado o estudo, que pode ter contribuído nos resultados encontrados. 
O penúltimo artigo traz um estudo qualitativo que apresenta a opinião dos pacientes de fisioterapia nas atividades desenvolvidas em seus tratamentos e sua percepção e participação baseada nessa reflexão.

\begin{tabular}{|c|c|}
\hline ANO & 2017 \\
\hline TÍTULO & $\begin{array}{c}\text { "In the physio w e trust": A qualitative study on patients' preferences for } \\
\text { physiotherapy }\end{array}$ \\
\hline AUTORES & $\begin{array}{l}\text { Susanne Bernhardsson } \\
\text { Maria E. H. Larsson } \\
\text { Kajsa Johansson } \\
\text { Birgitta Öberg }\end{array}$ \\
\hline OBJETIVO & $\begin{array}{c}\text { Criar conhecimento sobre as preferências dos métodos terapêuticos e } \\
\text { a participação na decisão das clínicas. }\end{array}$ \\
\hline MÉTODOS & $\begin{array}{l}\text { Foi feita uma pesquisa qualitativa de acordo com o COREQ com } 32 \\
\text { (trinta e dois) itens para estudos qualitativos. }\end{array}$ \\
\hline RESULTADOS & $\begin{array}{c}\text { A preferência por tratamentos ativos é relevante quanto a preferência } \\
\text { sobre outros aspectos. }\end{array}$ \\
\hline PUBLICAÇÃO & Physiotherapy Theory and Practice \\
\hline LOCAL & Gothenburg, Suécia \\
\hline
\end{tabular}

O artigo apresenta dados pautando a preferência dos pacientes mostrada a partir de seus pontos de vista e a sua relação com o produto proposto pelos guias de recomendação. Nos processos clinicais, cada vez mais, observa-se a incorporação destas opiniões mais frequentemente nas atividades práticas, a fim de promover bem-estar e interação com o usuário.

Uma vez que quanto maior for o conhecimento sobre a personalidade dos pacientes e suas minúcias, melhor pode ser o atendimento e ainda mais direcionado para o seu problema, funcionando como estímulo para um maior engajamento no tratamento de fisioterapia. Os pacientes submetidos aos checklist de estudos qualitativos foram recrutados nas proximidades das clínicas de fisioterapias de Gothenburg, na Suécia, com perfil socioeconômico variado. A entrevista era agendada e foi realizada com vinte participantes.

Um guia inicial foi desenvolvido pelo autor apresentando aspectos da experiência, preferência, expectativa e participação nas decisões pelo usuário. Os dados qualitativos foram analisados sistematicamente através das unidades, sentenças e parágrafos. Todos os elementos apresentados no estudo foram determinantes para as afirmativas anteriores.

Dos entrevistados, dez eram homens e dez mulheres com idade média de quarenta e oito anos. As profissões eram diversas, o nível de educação variava entre ensino médio e universidade e o tratamento de fisioterapia teve duração média de trinta meses para dezoito deles, sendo dois participantes neófitos.

Os pacientes abordaram que esperavam e preferiam predominantemente a experiência da fisioterapia baseada em exercícios, determinando que estes eram importantes para isolar o problema e encontrar a melhoria.

Também demonstraram maior comodidade com o agenciamento individual, ou seja, o tratamento singular. A acupuntura, a massagem relaxante e outros métodos passivos também foram apontados como preferidos.

A satisfação com experiências anteriores também contribui para experiências futuras, da mesma forma o desapontamento. Todos os participantes descreveram que gostariam de ter voz nas decisões clínicas durante o processo de tratamento. Não foi identificado nenhum estudo descrevendo a confiança na fisioterapia.

A experiência, a expectativa e satisfação são características que interferem diretamente na abordagem do paciente. $\mathrm{O}$ estudo sugere que eles devem ser contemplados nos tratamentos fisioterápicos a fim de estreitar a relação com o paciente e proporcionar um maior engajamento. 
O último artigo analisado foca na observação de centros de reabilitação para pacientes com paralisia cerebral e o processo de trabalho apresentado a partir da observação de seus coordenadores e funcionários.

\begin{tabular}{|c|c|}
\hline ANO & 2015 \\
\hline TÍTULO & $\begin{array}{c}\text { Work Processed Related to Cerebral Palsy of Neurological Rehabilitation } \\
\text { Centers }\end{array}$ \\
\hline AUTORES & $\begin{array}{c}\text { Ana Paula Mendonça } \\
\text { Shamyr Sulyvan Castro } \\
\text { John Henry Stone } \\
\text { Peterson M. O. Andrade }\end{array}$ \\
\hline OBJETIVO & $\begin{array}{l}\text { Avaliar o processo de trabalho nos centros de reabilitação baseados } \\
\text { nas recomendações da legislação brasileira. }\end{array}$ \\
\hline MÉTODOS & $\begin{array}{l}\text { Foi utilizado um instrumento de análise aplicado com os coordenadores } \\
\text { dos centros estudados. }\end{array}$ \\
\hline RESULTADOS & $\begin{array}{c}\text { Os centros de reabilitação no Brasil precisam integrar melhor as } \\
\text { necessidades das crianças e suas familias a partir do encontro primário } \\
\text { e do cuidado personalizado. }\end{array}$ \\
\hline PUBLICAÇÃO & Developmental Neurorehabilitation \\
\hline LOCAL & Minas Gerais, Brasil + Nova lorque, EUA \\
\hline
\end{tabular}

Este artigo tem como objetivo ser utilizado por profissionais de saúde assistencial no processo de reabilitação identificando forças e fraquezas no sistema de política de saúde do país, avaliando o processo de terapia da criança com PC nos centros de reabilitação do Brasil.

A reabilitação é um processo que promove a melhor qualidade de vida para os pacientes e familiares. Ela envolve um processo que é baseado na identificação individual do problema e necessidade específico dos enfermos. Como apresentado no trabalho, como passo inicial no tratamento desses pacientes se encontram os centros de reabilitação e os funcionários que desempenham todas as atividades.

O instrumento utilizado para avaliar os espaços em questão consistia em trinta e um itens com indagações sobre o processo de reabilitação sofrido pelos pacientes. Os primeiros dezoito foram classificados em: A - "'eu não sei dizer'” (0 pontos); B - "a ação não foi feita até então" (1 ponto);

C - "a ação foi feita, mas não teve continuidade no ano passado" (2 pontos);

D - "a ação foi feita, mas sem eficiência" (3 pontos);

E - "a ação foi feita, mas não houve percepção de melhoras na eficiência'" (4 pontos);

F - "a ação foi feita de forma eficiente, mas não houve melhora' (5 pontos);

$\mathrm{G}$ - "a ação foi feita de forma eficiente e sem nenhuma necessidade de alteração" (6 pontos).

Dos ambientes enquadrados como centros de reabilitação, quinze estavam presentes durante o "I Encontro de Network do Sistema de Saúde de Minas Gerais." Destes, dois representantes recusaram a participação na pesquisa. Portanto, no total havia treze estabelecimentos pesquisados. Os centros são regionais e promovem serviços para uma região específica do estado.

Considerando os dados observados, algumas ações consideradas de cunho obrigatório pela legislação vigente não são efetivadas pelos estabelecimentos. Por exemplo, o item relacionado à condução de atividades em grupo e visitas domiciliares é executado em apenas $40 \%$ desses centros.

A ação mais reportada pelos coordenadores como benéfica foi o encontro multidisciplinar sendo executado em todos os locais observados. Entretanto, ainda assim, o time de multidisciplinaridade se apresenta como 
inconsistente e fragmentado nos outros quesitos. Isso gera algumas incoerências vividas nesses pontos de assistência aos pacientes com paralisia cerebral, dificultando o processo de desenvolvimento da criança, ainda que em aspectos iniciais, pois sabe-se que as primeiras atitudes podem contribuir no quadro final do paciente com mais intensidade.

\section{Considerações Finais}

Os estudos que relacionam a adequação dos ambientes às atividades que são desenvolvidas em seu interior ainda são pouco expressivos. A relação humano-atividade-ambiente é fundamental na abordagem da Ergonomia do Ambiente Construído, sendo abordada em anos recentes, mas contando com limitadas publicações. Araújo et al (2016) apresentam um panorama das publicações em EAC no período de 2005 a 2015, em periódicos indexados no Qualis da CAPES, focando as oriundas de autores brasileiros e relacionando-os com os grupos de pesquisa e a existência de disciplina correlata nos cursos de arquitetura no país. Os resultados confirmam a produção não muito expressiva.

Os trabalhos que enfocam as atividades de atenção à saúde, raramente atentam às características do ambiente como facilitadoras para o trabalho dos profissionais. Conforme análise apresentada neste artigo, percebeu-se que as salas de fisioterapia para crianças com paralisia cerebral não têm sido exploradas sob a perspectiva da ergonomia do ambiente construído, especialmente no Brasil, como demonstra o último artigo apresentado. Os artigos relacionam a evolução da criança com paralisia cerebral à atividade fisioterapêutica, sendo desconsiderada a influência do ambiente sobre estes pacientes.

Na presente RSL, pode-se observar que as pesquisas voltadas para o campo de ambiente construído ainda se configuram de forma tímida, confirmando os achados de Araújo et al (2016) mesmo considerando os cinco anos passados desde a realização daquele trabalho. As pesquisas relacionadas ao tratamento fisioterapêutico de pacientes com PA superam sobremaneira os que tratam de ambientes, ainda que vários estudos apontem para a melhoria no quesito de saúde quando exploradas as características do espaço.

Neste contexto, poder-se-ia considerar a especificidade do tema tratado, no entanto, expandindo-se o escopo para ambientes de saúde abordados sob o foco da EAC, não são significativos os números encontrados.

Do contexto apresentado conclui-se que a lacuna identificada precisa ser sanada a partir do investimento em um aumento na investigação acerca do tema abordado, a fim de apresentar resultados significativos notadamente em periódicos internacionais qualificados.

\section{Referências Bibliográficas}

AGÊNCIA NACIONAL DE VIGILÂNCIA SANITÁRIA. RDC 50: Regulamento Técnico para planejamento, programação, elaboração e avaliação de projetos físicos de estabelecimentos assistenciais de saúde. Brasília: ANVISA, 2002.

ANABY, D.; LAW, M.; TEPLICKY, R.; TURNER, L. Focusing on the Environment to Improve Youth Participation: Experiences and Perspectives of Occupational Therapists. Canadá: International Journal of Environmental Research and Public Health, 2015. DOI: 10.3390/ijerph121013388

ARAÚJO, Maiana C., CAMPOS, Fábio, VILLAROUCO, Vilma. Cenário da produção científica brasileira sobre Ergonomia do Ambiente Construído (2005-2015). In: MONT'ALVÃO, Cláudia; VILLAROUCO, Vilma. (Orgs). Um novo olhar para o projeto: a ergonomia no ambiente construído - Volume 3. Editora Livro Rápido: Olinda, PE, 2016. 
ASSOCIAÇÃO BRASILEIRA DE NORMAS TÉCNICAS. NBR 6.022: Artigos científicos impressos. Rio de Janeiro: ABNT, 2018.

BAQAI, A.; MEMON, K.; MEMON, A. R.; SHAH, S. M. Z. A. Interactive Physiotherapy: An Application Based on Virtual Reality and Bio-feedback. Paquistão: Cross Mark, 2018. DOI: https://doi.org/10.1007/s11277-018-5382-5

BERNHARDSSON, S.; LARSSON, M. E. H.; JOHANSSON, K.; ÖBERG, B. "In the physio we trust": A qualitative study on patients' preferences for physiotherapy. Suécia: Physiotherapy Theory and Practice, 2017. DOI: https://doi.org/10.1080/09593985.2017.1328720

Brasil. Ministério da Saúde. Secretaria de Assistência à Saúde. Normas para projetos físicos de estabelecimentos assistenciais de saúde. Brasília: Coordenação-Geral de Normas,1994.

CLARK, C.; SLIKER, L. SANDSTRUM, J.; BURNE, B.; HAGGETT, V.; BODINE, C. Development and Preliminary Investigation of a Semiautonomous Socially Assistive Robot (SAR) Designed to Elicit Communication, Motor Skills, Emotion, and Visual Regard (Engagement) from Young Children with Complex Cerebral Palsy: A Pilot Comparative Trial. Estados Unidos: Hindawi Advances in HumanComputer Interaction, 2019. DOI: https://doi.org/10.1155/2019/2614060

FERRARETTO, I.; SOUZA, A. M. C. Paralisia Cerebral - aspectos práticos. São Paulo: Memnon, 1998.

GUNATILLAKA, K. A. N. Cerebral palsy - understanding the disabilities and planning intervention. Sri Lanka: Journal of Child Health, 33. https://doi.org/10.4038/sljch.v33i2.652

GUPTA, R.; APPLETON, R. E. Cerebral palsy: not always what it seems. Reino Unido: Archives of Disease in Childhood, 2001.

IIDA, Itiro. Ergonomia: projeto e produção. São Paulo: Editora Blucher, 2005.

MENDONÇA, A. P.; CASTRO, S. S.; STONE, J. H.; ANDRADE P. M. O. Work process related to cerebral palsy of neurological rehabilitation centers. Reino Unido: Informa Health Care, 2018. DOI: $10.3109 / 17518423.2013 .796418$

MONT'ALVÃO, Claudia; VILLAROUCO, Vilma (Org.) . Um novo olhar para o projeto 4: a ergonomia no ambiente construído. 1. ed. Olinda, PE: Livro Rápido, 2018. v. 4. 372p.

RATLIFFE, K. T. Fisioterapia na clínica pediátrica. $1^{\text {a }}$ ed. São Paulo: Santos, 2000.

ROSSA, S. M.; CASE, L., LEUNGA, W. Aligning Physical Activity Measures With the International Classification of Functioning, Disability and Health Framework for Childhood Disability. Estados Unidos: Quest, 2016. DOI: http://dx.doi.org/10.1080/00336297.2016.1145128

SCHUMANN, L.; BOIVIN, M.; PAQUIN, S.; LACOURSE, E.; BRENDGEN, M.; VITARO, F.; DIONNE, G.; TREMBLAY, R. E.; BOOI, L. Persistence and innovation effects in genetic and environmental factors in negative emotionality during infancy: A twin study. Canadá: PLoS ONE, 2017. DOI: https://doi.org/10.1371/journal.pone.0176601

VASCONCELOS, C. F.; VILLAROUCO, V.; SOARES, M. M. Contribuição da psicologia ambiental na análise ergonômica do ambiente construído. Recife: Ação Ergonômica, 2010. Disponível em: $<$ http://www.abergo.org.br/revista/index.php/ae/article/view/92>. Acesso em: 30 ago. 2020. 
VILLAROUCO, V. O que é um ambiente ergonomicamente adequado? São Paulo: I Conferência LatinoAmericana de construção sustentável, 2004. Disponível em: <ftp://ip20017719.eng.ufjf.br/Public/AnaisEventosCientificos/ENTAC_2004/trabalhos/PAP007 0d.pdf>. Acesso em: 30 ago. 2020.

\section{Agradecimentos}

As autoras deste artigo agradecem ao CNPq e ao Programa de Pós-Graduação em Design da UFPE pela contribuição recebida para o desenvolvimento deste trabalho. 